\title{
Impact of the COVID-19 pandemic in nuclear medicine departments: preliminary report of the first international survey
}

\author{
Salvatore Annunziata ${ }^{1} \cdot$ Matteo Bauckneht ${ }^{2}$ - Domenico Albano ${ }^{3}$ - Giovanni Argiroffi ${ }^{4}$ - Diletta Calabrò ${ }^{5}$. \\ Elisabetta Abenavoli ${ }^{6}$. Flavia Linguanti ${ }^{6} \cdot$ Riccardo Laudicella $^{7}$ - on behalf of the Young Committee of the Italian \\ Association of Nuclear Medicine (AIMN)
}

Received: 22 April 2020 / Accepted: 18 May 2020 / Published online: 27 May 2020

(C) Springer-Verlag GmbH Germany, part of Springer Nature 2020

\begin{abstract}
Purpose Coronavirus disease-19 (COVID-19) pandemic is challenging the availability of hospital resources worldwide. The Young Group of the Italian Association of Nuclear Medicine (AIMN) developed the first international survey to evaluate the impact of COVID-19 in nuclear medicine (NM). The aim of this study was to perform a preliminary report of the ongoing survey. Methods A questionnaire of thirty questions was prepared for all NM professionals addressing three main issues: (1) new scheduling praxes for NM diagnostic and therapeutic procedures, (2) assistance of patients with diagnosed or suspected COVID-19, and (3) prevention of COVID-19 spreading in the departments. An invitation to the survey was sent to the corresponding authors of NM scientific papers indexed in SCOPUS in 2019. Personal data were analysed per individual responder. Organisation data were evaluated per single department.

Results Two-hundred and ninety-six individual responders from 220 departments were evaluated. Most of the responders were from Europe (199/296, 67\%). Approximately, all departments already changed their scheduling praxes due to the pandemic (213/ $220,97 \%)$. In most departments, scheduled diagnostic and therapeutic procedures were allowed but quantitatively reduced (112/ 220,51\%). A significant reduction of diagnostic and therapeutic procedures (more than 20\%) affected 198/220 (90\%) and 158/ $220(72 \%)$ departments, respectively. Incidental COVID-19 signs in NM exams occurred in 106/220 departments (48\%). Few departments were closed or shifted to assist patients with COVID-19 (36/220,16\%). Most of the responders thought that pandemic would not permanently change the work of NM departments in the future (189/296, 64\%).

Conclusions According to this preliminary report of the first international survey, COVID-19 heavily impacted NM departments and professionals. New praxes for NM procedures, assistance, and prevention of COVID-19 have been applied during the pandemic.
\end{abstract}

Keywords COVID-19 $\cdot$ Nuclear medicine $\cdot$ Survey

This article is part of the Topical Collection on Infection and inflammation.

Salvatore Annunziata

salvatoreannunziata@live.it

1 Nuclear Medicine Unit, IRCCS Regina Elena National Cancer Institute, via Elio Chianesi 53, 00144 Rome, Italy

2 Nuclear Medicine Unit, IRCCS Ospedale Policlinico San Martino, Genoa, Italy

3 Department of Nuclear Medicine, University of Brescia and Spedali Civili Brescia, Brescia, Italy
4 Department of Health Sciences, University of Milan, Milan, Italy

5 Nuclear Medicine, DIMES University of Bologna, S. Orsola-Malpighi Hospital, Bologna, Italy

6 Nuclear Medicine Unit, Department of Experimental and Clinical Biomedical Sciences "Mario Serio", University of Florence, Florence, Italy

7 Department of Biomedical and Dental Sciences and of Morpho-Functional Imaging, Nuclear Medicine Unit, University of Messina, Messina, Italy 


\section{Introduction}

Coronavirus disease-19 (COVID-19) is an infectious acute respiratory syndrome caused by the severe acute respiratory syndrome coronavirus 2 (SARS-CoV-2) [1-3]. COVID-19 mainly affects the lower respiratory tract and causes flu-like symptoms. In severe cases, COVID-19 determines interstitial pneumonia that may evolve in acute respiratory distress syndrome and death. The diagnosis is made by performing a test on patients' biological samples [1-3]. Nowadays, there is no specific vaccine or treatment for this disease: currently, the treatment consists of isolating the patients and managing their clinical symptoms. In most cases, the spread between people occurs through the respiratory droplets emitted by coughing or sneezing [1-3]. To limit the transmission, precautions must be endorsed, such as avoid interpersonal and tight contacts, adopting careful personal hygiene, washing hands frequently, and wearing masks. Namely, a person who is not equipped with the appropriate personal protective equipment (PPE) will be exposed to contact, becoming potentially contagious to other people even if asymptomatic [1-3].

During this COVID-19 pandemic, nuclear medicine (NM) departments could have been involved in changes and issues, such as new scheduling praxes for NM diagnostic and therapeutic procedures, assistance of patients with diagnosed or suspected COVID-19, and prevention of COVID-19 spreading in the departments [4-23]. COVID-19 patients are usually assessed with radiological investigations, and high-resolution chest computer tomography (CT) is the preferred tool. However, also NM personnel is at risk for exposure to the virus. Several authors showed an ever-growing incidental pulmonary picture suggestive of COVID-19, highlighted in the CT component of the predominantly oncological positron emission tomography (PET)/CT studies [4-15]. For this reason, first recommendations and comments have been recently published to help NM professionals in their daily workflow during the pandemic [16-23].

According to a standardised methodology and to evaluate the different experiences of NM professionals in the international scenario, the Young Group of the Italian Association of NM (AIMN) recently developed the first international survey about the impact of COVID-19 in NM departments worldwide [24-31]. This study aims to perform a preliminary report of the ongoing survey.

\section{Methods}

\section{Survey preparation}

A web-questionnaire was prepared by the Young AIMN Group to evaluate the impact of COVID-19 pandemic in NM departments, in line with recent recommendations and requirements to maximise response rates such as a personal introductory statement, the offer to make results public, the use of simple headers and textual representation of response categories, and the prerequisite of a relatively short deadline including multiple reminders [24-31].

Thirty questions in the English language were prepared for all healthcare professionals of NM departments worldwide (Appendix). Question types were dichotomous, single-choice, multiple-choice, rating scales, or open-ended for number, text comment, or date [24-31]. Preliminary questions about personal and demographic data were included. Subsequently, questions about the COVID-19 pandemic were prepared to address three main issues: (1) new scheduling praxes for diagnostic and therapeutic procedures, (2) assistance of patients with diagnosed or suspected COVID-19, and (3) prevention of COVID-19 spreading in the departments. Finally, some questions about further development after the end of the emergency were proposed.

\section{Survey invitations}

The questionnaire was placed in Google Form Document (Appendix). Since the 1st of April 2020, an invitation to the survey was sent to the corresponding authors of scientific papers in the field of NM indexed in SCOPUS in 2019, using the following query string: ALL ("nuclear medicine") SUBJAREA (MEDI). Two reminders were mailed to all non-responding recipients. To further increase potential responses, the survey weblink was accessible by electronic devices and social media.

\section{Data collection and analysis}

For this preliminary report, the responses received until the 20th of April 2020 were evaluated anonymously. All responses were checked for completeness and collected in a Microsoft Excel table. Personal and demographic data were analysed per individual responder. Organisation and assistance data were evaluated per single department. When more than one response was received from the same department, only the response from the apical figure (e.g. the chief) was considered about the organisation data. To perform a preliminary report of this survey, we reported the total number of responses per answer option, the proportion of responses per respective answer option in percent, the rank of answer options, or median (range) for a quantitative response [24-31]. Free text responses were summarised into categories.

\section{Results}

\section{Demographic data}

Two-hundred and ninety-six individual responses from 220 departments were evaluated in this preliminary report (Fig. 1). Most of the responders were corresponding authors of NM paper 


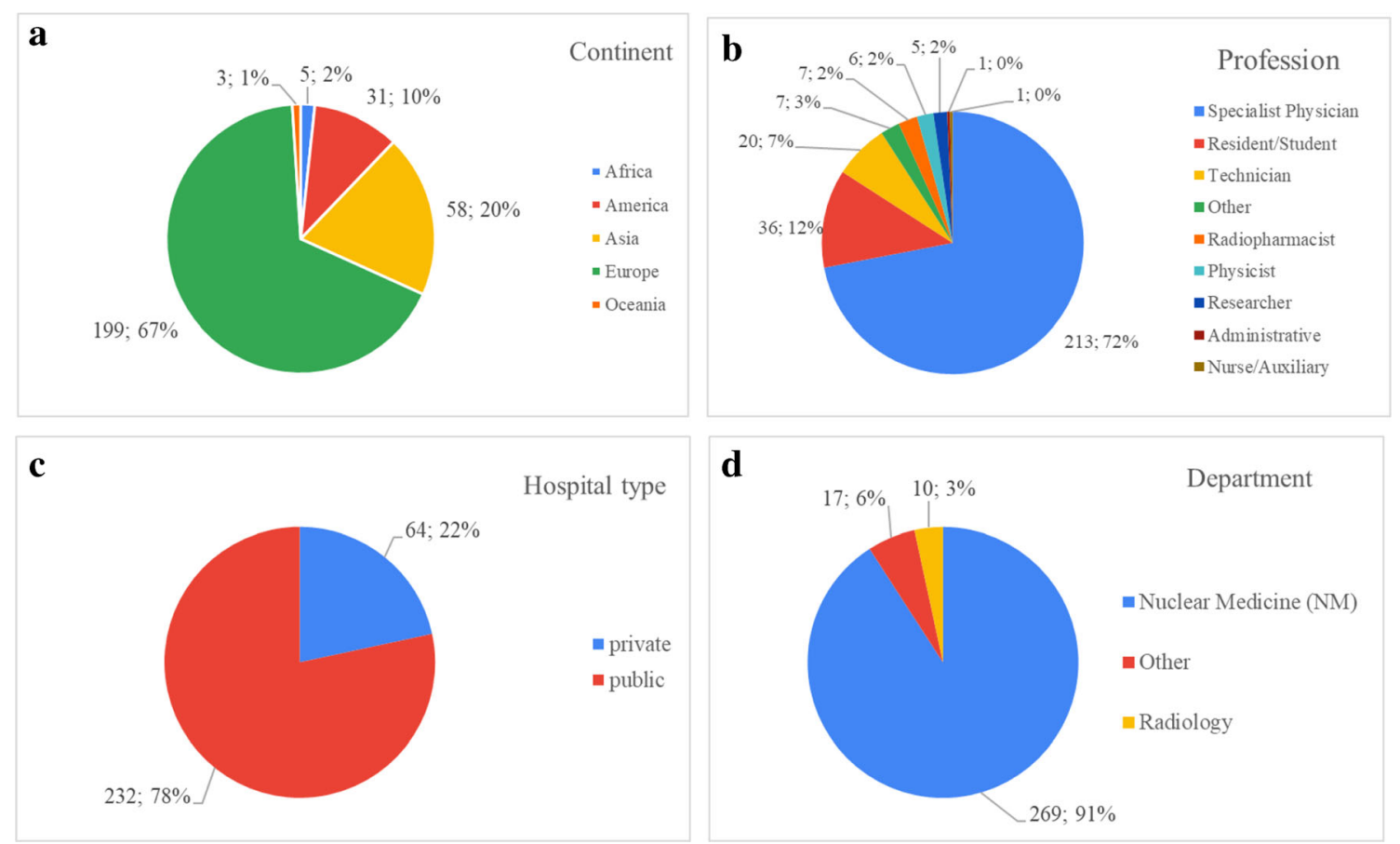

Fig. 1 Demographic data about the responders to the survey, such as continent of origin (a), profession (b), hospital type (c), and department (d)

indexed in SCOPUS in 2019 (270/296, 91\%). The mean age of responders was of 42 years (range 22-69). Most of the responders were from Europe (199/296, 67\%). Italy was the prevailing nation among responders $(89 / 296,30 \%)$ and departments (41/220, 18\%), respectively. Most of the responders were NM specialist physicians $(213 / 296,72 \%)$, from public hospitals (232/ 296, 78\%). Approximately, all responders were from NM departments: only a few responders were from radiology departments or other departments (e.g. cardiology, Fig. 1). Most of the departments were equipped with the main NM facilities such as singlephoton emission tomography (SPET), PET, and therapy ward.

\section{Scheduling of NM procedures}

At the time of the response, approximately all departments already changed their scheduling workflow due to the pandemic (213/220, 97\%, Fig. 2). Scheduled diagnostic and therapeutic procedures were allowed but quantitatively reduced in half of the departments $(112 / 220,51 \%)$. A significant reduction of diagnostic and therapeutic procedures (more than 20\%) affected 198/220 (90\%) and 158/220 (72\%) departments, respectively. Conventional scintigraphy/SPET sections seemed to be the most affected $(155 / 220,70 \%)$. Nuclear cardiology examinations showed a slight prevalence in the quantitative reduction among the diagnostic procedures $(58 / 220,26 \%)$, but neurology and oncology were also affected. Radioiodine therapy seemed to have the most evident reduction among the therapy procedures $(126 / 220,57 \%)$. A quote of the departments experienced a delay in radiopharmaceuticals supply or technical assistance during the pandemic $(92 / 220,42 \%)$.

\section{Assistance of patients with COVID-19 or suspected symptoms}

Patients with COVID-19 or suspected symptoms (e.g. cough, fever) were evaluated for possible NM procedures by both phone and on-site pre-triage in approximately all departments. When on-site triage was possible, clinical evaluation and thermal scanner were used in 183/220 (83\%) and 132/220 (60\%) departments, respectively.

About the COVID-19 prevalence among NM patients (Fig. 3), 65/220 departments (30\%) experienced at least one patient with COVID-19 until the 31st of March 2020, while 100/220 departments (45\%) are expected to assist at least one patient with COVID-19 until the 31 st of May 2020. A similar trend emerged for patients with suspected clinical symptoms. Incidental COVID-19 signs in NM exams occurred in 106/220 departments (48\%). Few departments were closed or shifted to directly assist patients with COVID-19 (36/220, 16\%). In some departments, professionals from NM were shifted part-time or full-time to the assistance of patients with COVID-19 (76/ $220,44 \%)$. Few departments were involved in the screening of COVID-19 by using low-dose CT integrated into SPET/PET tomographs $(23 / 220,10 \%)$. In an open question, NM examinations were not considered helpful in the management of COVID-19 by 99/296 responders (33\%), even though 18F-fluorodeoxyglucose (FDG) PET/CT and perfusion lung scan were often mentioned as possible diagnostic tools. 

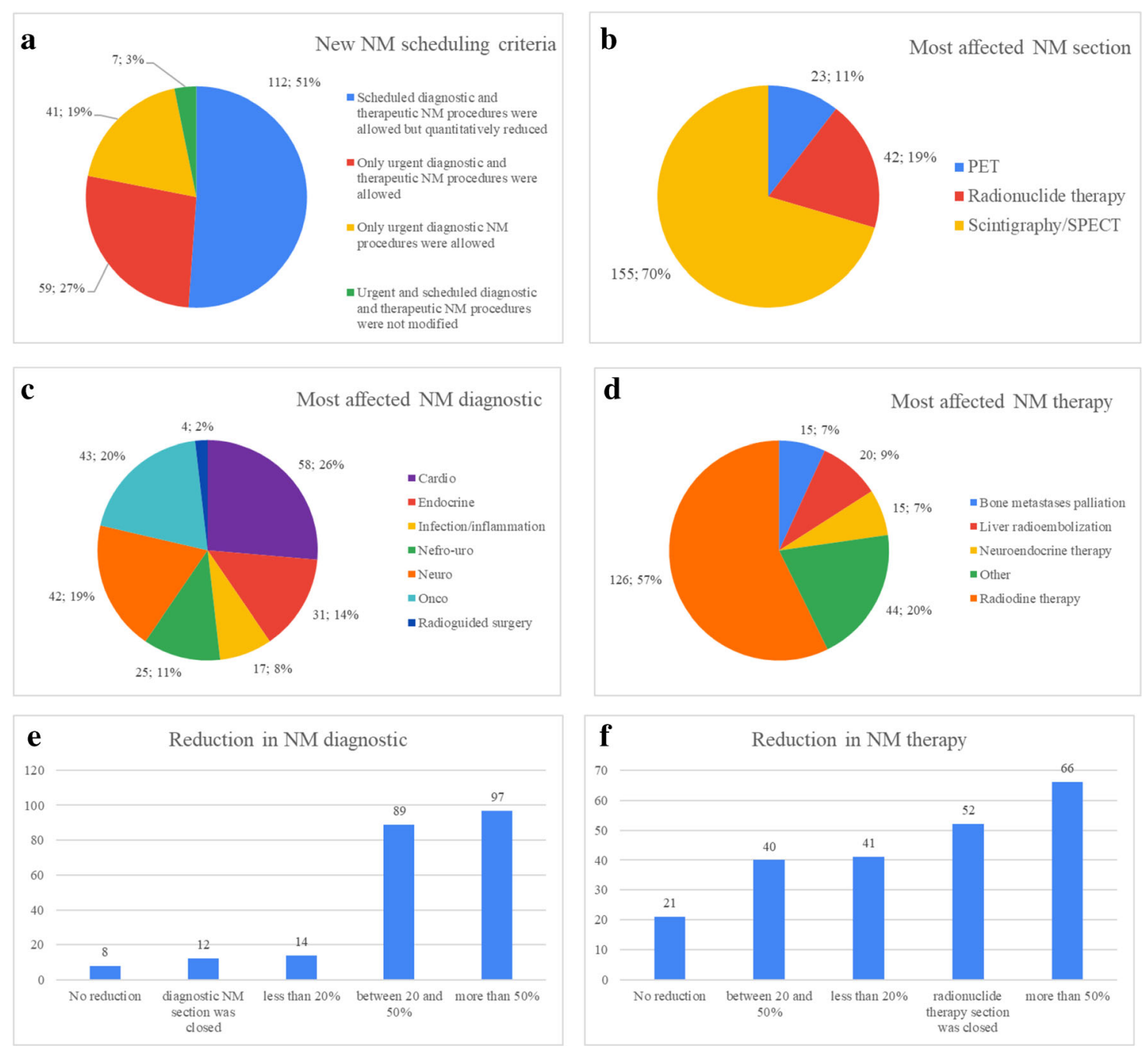

Fig. 2 Data about new criteria for NM procedures during the pandemic, such as new scheduling praxes (a), most affected NM sections (b), diagnostic areas (c) and radionuclide therapies (d), quantitative

\section{Prevention of COVID-19 spreading in NM departments}

Several departments ensured proper distancing and PPE for NM staff and patients $(200 / 220,91 \%)$, such as surgical masks and gloves. Namely, 191/220 departments (87\%) used surgical masks for all NM professionals, and 141/220 departments (64\%) used surgical masks for all patients. At the time of the response, at least one NM physician and other healthcare professional had a diagnosis of COVID-19 in 54/220 (23\%) and 80/220 (36\%) departments, respectively (Fig. 3).

\section{Further perspectives}

Finally, 189/296 responders (64\%) thought that pandemic would not permanently change the work of NM departments in the future (Fig. 4). Most of the responders thought that the emergency status in NM due to the pandemic could end until

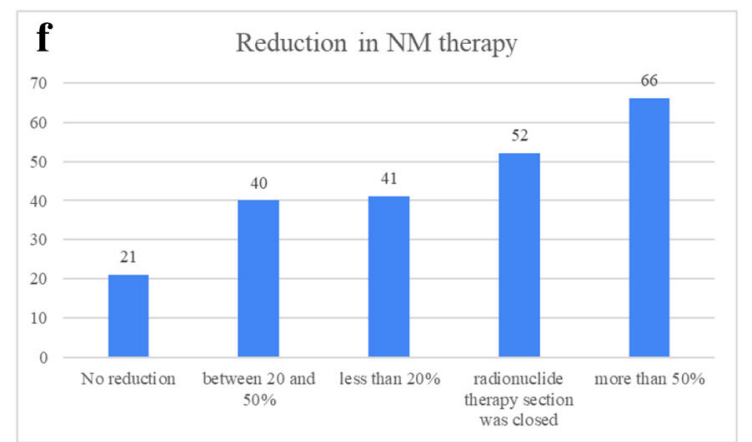

reduction of NM diagnostic examinations (e), and radionuclide therapies (f)

June 2020. In an open question, the responders expressed their opinion about further changes in NM departments after the end of the emergency: when affirmative, prevalent responses were "implementation of hygienic rules" and "reduction of inappropriate examinations" (Fig. 4).

Since Italy was the prevalent nation among responders and departments $(41 / 220,18 \%)$, an overview of the main responses by the Italian departments is shown in Fig. 5. Italian findings were concordant with international evidence about new scheduling praxes, assistance of patients, and prevention of COVID-19 spreading.

\section{Discussion}

The COVID-19 pandemic had a significant impact on hospital resource and organisation worldwide [1-3]. Several hospital departments shifted their work to the management of COVID- 

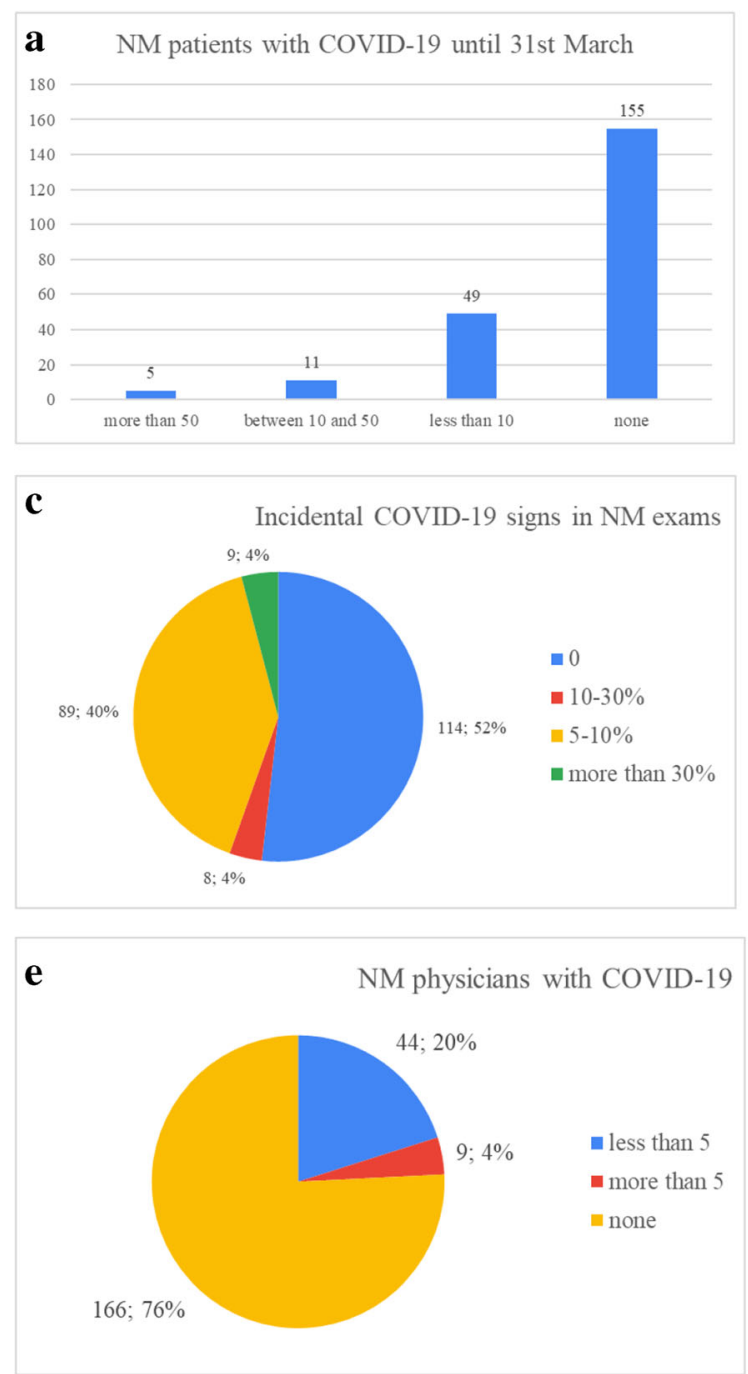

Fig. 3 Data about COVID-19 assistance and prevention, such as the number of patients with COVID-19 to 31st March and 31st May 2020 (a, b), NM exams showing COVID-19 signs (c), departments closed or

19 patients. The Young AIMN Group prepared the first international survey to appraise the different experiences of NM physicians and other healthcare professionals in their work. The aim of the present study was to perform a preliminary report of this first international survey.

About the demographic data, most of the responders were specialist physicians, even though a number of other healthcare professionals participated as well (e.g. residents, technicians). In fact, we decided to make the survey available to all professionals who work in NM departments, in order to have a comprehensive overview of the current situation. According to the overall number of publications in the field of NM in 2019, a large number of responders were from Europe. In fact, the survey was sent to all corresponding authors indexed on SCOPUS who published in the field of NM in 2019, in order to have a systematic criterion for inviting authors. Private/public hospitals as well as NM facilities
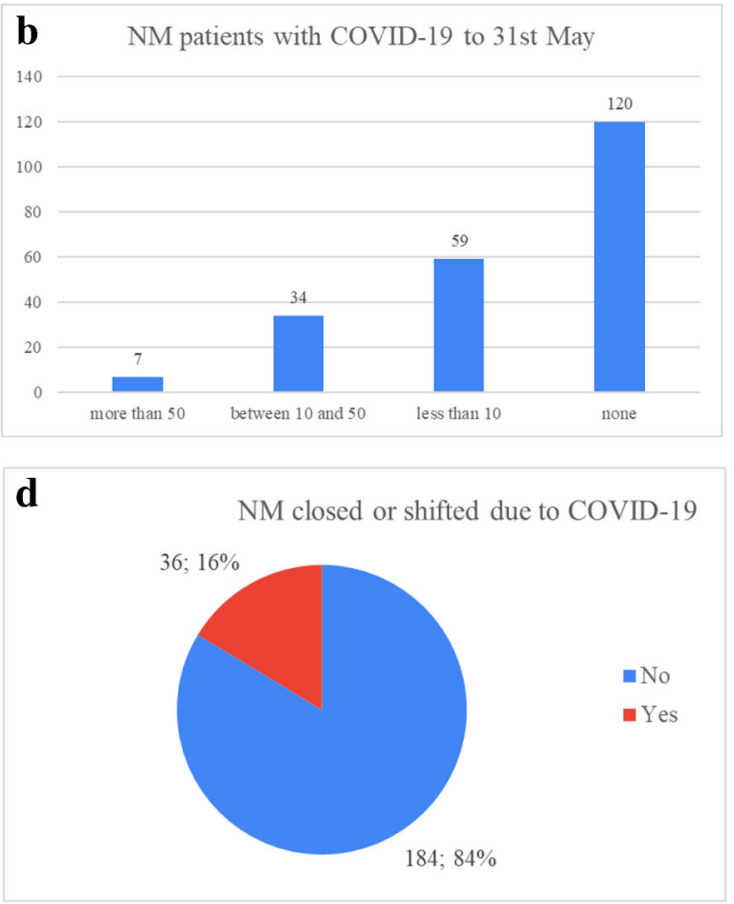

f

Other NM professionals with COVID-19

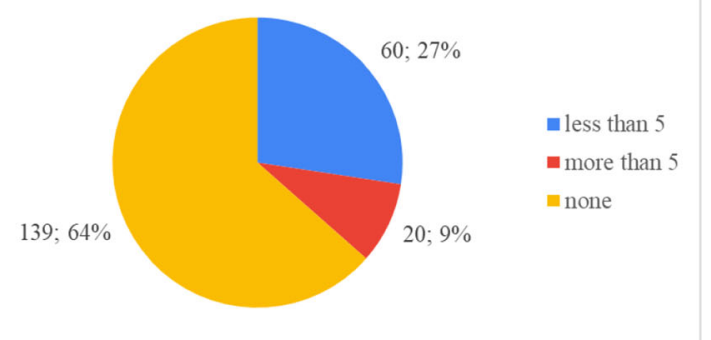

shifted to COVID-19 assistance (d), NM physicians and other professionals with COVID-19 (e, f)

(scintigraphy/SPET, PET, and therapy ward) were equally represented among the included departments.

About new scheduling praxes for NM procedures, the COVID-19 pandemic already impacted in approximately all departments, where diagnostic and therapeutic procedures were often allowed but quantitatively reduced. These data are in line with suggestions and reports recently published by the main scientific societies and journals in our field [16-23]. Conventional gamma-camera sections seemed to be more affected by the reduction than PET and radionuclide therapy sections. In accordance with a recent local survey [32], NM diagnostic exams in the areas of cardiology, neurology, and oncology were all affected by a quantitative reduction. A possible explanation is that often only urgent examinations were allowed in several hospitals; therefore, some deferrable exams could have been postponed. At the same time, radioiodine therapy had the most evident quantitative 


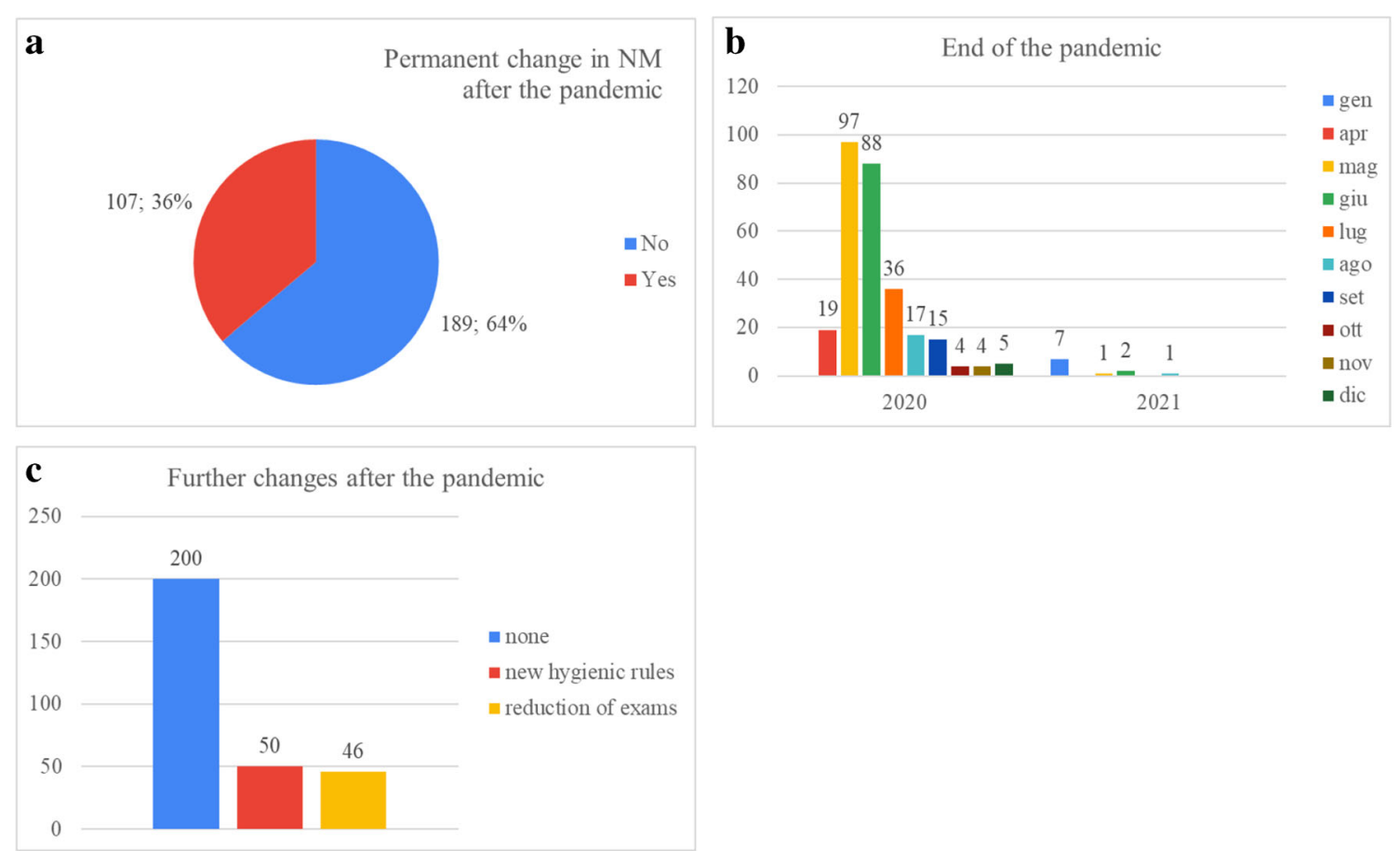

Fig. 4 Data about further perspectives, such as possible permanent changes after the pandemic (a), opinion about the end date of the pandemic (b), and open responses about further changes in NM departments (c)

reduction among the therapy sections. Considering the good outcome survival and prognosis of the patients affected by differentiated thyroid cancer, the radioiodine therapy after surgery may have been postponed without significantly influencing the management, differently from other radiometabolic therapies performed for more aggressive oncological diseases (e.g. bone palliation, liver radioembolisation, and peptide receptor radionuclide therapy). Interestingly, about half of the departments experienced a delay in radiopharmaceuticals supply or technical assistance during the pandemic, with possible further discomforts for NM professionals and patients.

About the assistance of patients with COVID-19 or suspected clinical symptoms (e.g. cough, fever), most of the departments received new indications and restrictions by each hospital. In particular, phone and on-site pre-triage were often implemented to evaluate the patients with active COVID-19 or suspected clinical symptoms eventually candidate for NM examinations and therapies. Some differences could be found among the on-site triage tools (e.g. clinical evaluation or thermal scanner), probably due to different hospital resources and pandemic stages. According to our preliminary report, only a quote of departments experienced patients with COVID-19 until the 31 st of March 2020 or expect to assist patients with COVID-19 until the 31st of May 2020. Nevertheless, different COVID-19 prevalence and pandemic stages among countries might be considered. At the moment, when the assistance of patients with COVID-19 or suspected symptoms is required, a robust screening process for outpatients should be followed as suggested by main recent recommendations [16-23].
Accordingly, some departments found incidental COVID19 signs in routinary NM examinations, as recently described by some case reports or series [4-16]. Only a few NM departments or professionals were shifted to directly assist patients with COVID-19 or involved in the screening of COVID-19 by using low-dose CT integrated into SPET/ PET tomographs. Those interventions could have been implemented in some high-prevalence regions for the time of the emergency. Interestingly, some responders thought that no NM examinations can be useful in the ordinary management of COVID-19, even though FDG-PET/CT and perfusion lung scans were often mentioned. Accordingly, recent studies evaluated the possible role of FDG-PET/CT and perfusion lung scan in the management of COVID-19 pneumonia, to evaluate the entity of inflammatory and embolic pattern respectively [4-16]. Nevertheless, the role of NM examinations in the ordinary management of COVID-19 is still unclear [33]. At the moment, recent studies only showed incidental or rare findings of COVID-19 in NM examinations. Further studies are needed in order to define the exact role of FDG-PET/CT, perfusion lung scan, integrated lowdose CT, or new NM tools in patients with COVID-19 [33]. Nevertheless, when a suspicion of incidental COVID19 findings raises by NM exams, prompt communication to dedicate offices should be performed according to recent recommendations [16-23]. Probably, a particular attention to incidental lung findings in routinary SPET/CT and PET/CT could be suggested during the pandemic period. This praxis could correctly address suspected patients to the ordinary 

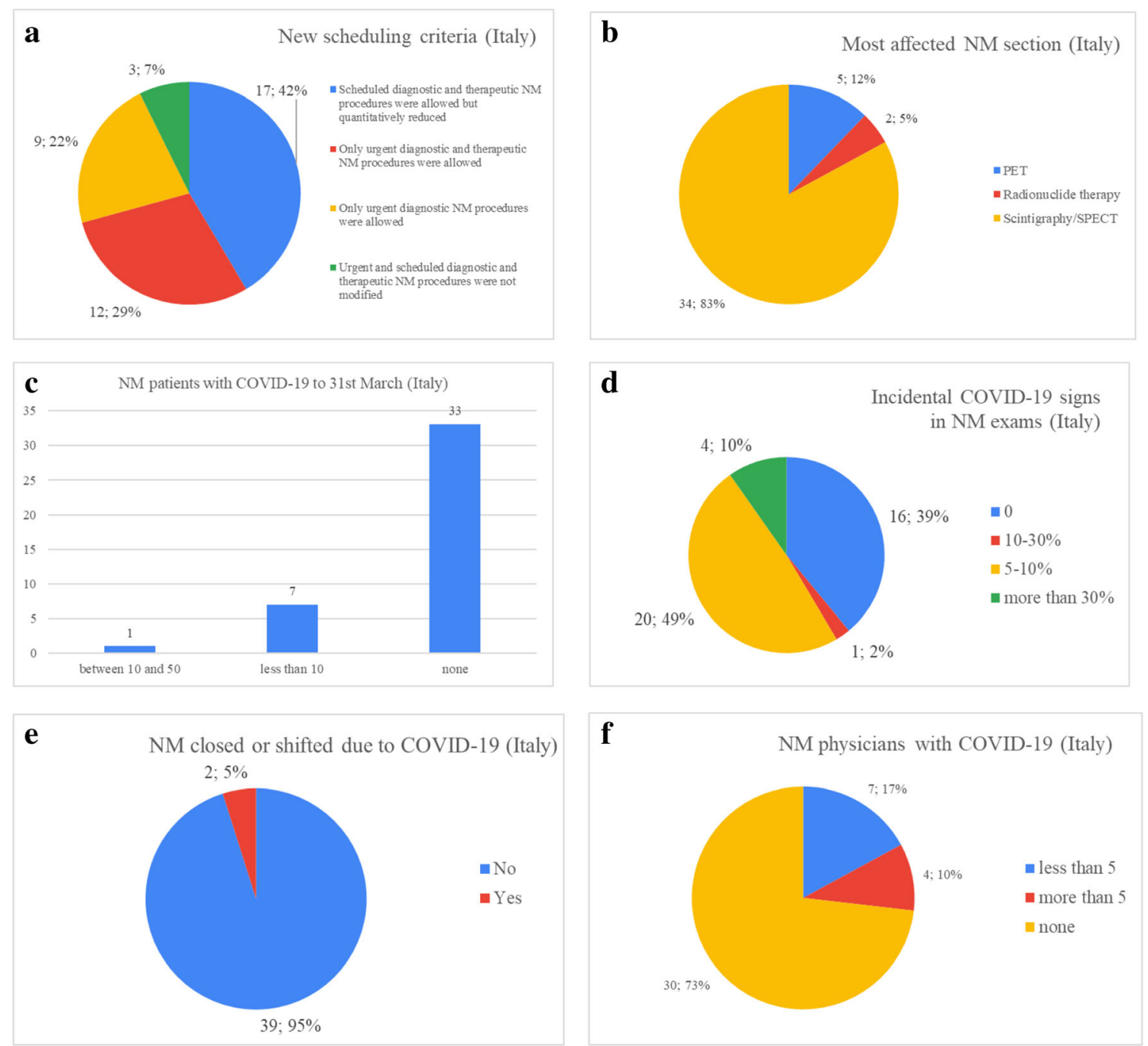

Fig. 5 Overview of the main responses from the Italian departments (41/220, 18\%), such as new scheduling praxes (a), most affected NM section (b), NM patients with COVID-19 (c), incidental COVID-19 signs (d), NM closed or shifted (e), NM physicians with COVID-19 (f)

screening tools of COVID-19 (e.g. clinical evaluation, swabs) and to prevent undesirable spreading of COVID-19 within the departments.

About the prevention of COVID-19 spreading, most of the departments seemed to offer proper PPE to their staff. Surgical masks were worldwide diffused for professionals and patients, as recommended by recent publications and scientific societies [16-23]. Gloves, eye protection, and other equipment could be available in particular situations. As well known, the risk of spreading of COVID-19 in hospitals and NM departments could exist [16-23]: according to this preliminary report, some NM physicians and healthcare professionals had a diagnosis of COVID-19 at the time of response. Nevertheless, the use of proper PPE should reduce the risk of spreading and ensure the health of all professionals who work in NM departments. At the moment, a clear escalation pathway for COVID-19 cases and the use of severe hygienic rules should be guaranteed in all departments, as suggested in recent guidelines [16-23].
About further perspectives, most of the professionals thought that the pandemic would not permanently change the work of NM departments in the future, and several responders thought that the pandemic could end in the next weeks. Nevertheless, new hygienic rules such as distancing, handwashing, and routinary use of surgical masks emerged as useful tools to be pursued also after the end of the emergency. Possible solutions to ensure distancing such as new staff tournaments and new praxes for patients' appointments should be evaluated. Moreover, some responders proposed a deeper evaluation of the appropriateness and the urgency of NM exams. To this aim, an evidence-based approach could help to critically appraise the impact and the timing of NM procedures and to eventually improve the organisation of NM departments during and after the end of the pandemic [33]. Furthermore, the role of scientific societies might be crucial in the national and international scenario to propose and renew specific guidelines about all the organisational aspects of NM departments during and after the emergency. 
This study has some limitations. This is a preliminary report of the first international survey about the impact of COVID-19 in NM; therefore, further studies will be performed in order to deeper analyse complete data of the survey. According to previous methodologies [24-31], corresponding authors of NM scientific papers indexed in SCOPUS in 2019 received an invitation to the survey. When more than one response was received from the same department, only the response from the apical figure was considered about the organisation of the department. We are aware that the epidemic curve and the department organisation may differ among countries, so deeper sub-analyses according to geographical criteria will be performed in the subsequent studies, as well as sub-analyses according to other demographic criteria (e.g. healthcare profession). Since Italy was the prevalent nation among responders and departments, an overview of the responses from Italy was performed in this preliminary report.

\section{Conclusion and suggestions}

According to this preliminary report of the first international survey, COVID-19 heavily impacted NM departments and healthcare professionals. New workflow organisation for NM procedures has been applied during the pandemic, as well as for the assistance and the prevention of COVID-19. Possible differences in geographical and professional subgroups might be further analysed.

The following suggestions for future actions could be derived from this study:

- All NM professionals should be aware of COVID-19 risks and informed by guidelines and scientific societies about the pandemic stage and the organisational rules within the departments.

- NM examinations should be scheduled following the local and general recommendations about the COVID-19 emergency, as well as urgency and appropriateness criteria.

- A robust screening process such as phone or on-site pretriage should be performed in NM departments before scheduling patients for NM procedures.

- NM professionals and patients should strictly follow the hygienic rules provided by recent guidelines to prevent the COVID-19 spreading within the departments, such as proper distancing and prompt escalation for suspected cases.

- Further investigations are needed to generate clear evidence for possible indications of NM examinations in the ordinary management of COVID- 19 .

Acknowledgments We are grateful to all the responders of this survey for their participation and support.

\section{Compliance with ethical standards}

Conflict of interest The authors declare that they have no conflict of interest.

Ethical approval All procedures performed in studies involving human participants were in accordance with the ethical standards of the institutional and/or national research committee and with the 1964 Helsinki Declaration and its later amendments or comparable ethical standards.

Informed consent Informed consent to anonymously collect and publish personal and opinion data of this survey was obtained in the presentation page of the web questionnaire.

\section{Appendix Questionnaire}

\section{Demographic data}

Name and surname; Age; Healthcare profession; Department; Continent; Nation; Region/State; City; Hospital name; Hospital type; Personal introductory statement; How do you look for information about the impact of COVID-19 pandemic in your daily work?

\section{New criteria for NM procedures}

- What kind of NM facilities do you have in your department?

- Has COVID-19 pandemic already changed scheduling criteria in your NM department?

- Which scheduling criteria are you applying in your NM department during COVID-19 pandemic?

- In your experience, which section of your NM department has been more affected by new criteria?

- In your experience, which clinical area of NM diagnostic exams has been more affected by new criteria?

- In your experience, what kind of radionuclide therapy has been more affected by new criteria?

- Can you quantify the reduction of diagnostic exams in your Department?

- Can you quantify the reduction of radionuclide therapies in your Department?

- Did you have any delay or problems for radiopharmaceuticals supply or technical assistance due to the pandemic emergency?

\section{Assistance of patients with COVID-19 or suspected clinical symptoms}

- Which indications about assistance of certain COVID-19 patients did you receive by your hospital?

- Which operative procedures do you follow to assist patients with suspected clinical symptoms? 
- If on-site triage is possible, what kind of data or exams are available for your evaluation?

- How many patients with certain COVID-19 diagnosis did you assist up to 31st March 2020?

- How many patients with suspected clinical symptoms did you assist up to 31st March 2020?

- How many patients with certain COVID-19 do you plan to assist up to 31st May 2020?

- How many patients with suspected clinical symptoms do you plan assist up to 31st May 2020?

- In your opinion, which new or old NM examinations might help the management of COVID-19?

- How many patients did show COVID-19 signs in scintigraphy, SPECT-CT or PET-CT (\%)?

- Did your NM department have been closed or shifted to directly assist COVID-19 patients?

- Did you or NM staff have been shifted part-time or fulltime in a COVID-19 unit?

- Has your department been involved in COVID-19 screening by low-dose CT of SPET/PET-CT tomographs?

\section{Prevention of COVID-19 spreading}

- Which procedures do you follow to prevent COVID-19 spreading?

- To your knowledge, how many physicians had COVID19 in your Department?

- To your knowledge, how many other healthcare professionals had COVID-19 in your Department?

- What kind of personal protective equipment is available in your department?

- What kind of personal protective equipment do your patients use during pandemic?

- What kind of personal protective equipment do you and your staff use during pandemic?

\section{Further perspectives}

- Will COVID-19 pandemic permanently change scheduling criteria in your Nuclear Medicine department?

- In your opinion, when the emergency status in your NM departments due to pandemic will end?

- How COVID-19 pandemic will change in the future our work in Nuclear Medicine departments?

\section{References}

1. Huang C, Wang Y, Li X, Ren L, Zhao J, Hu Y, et al. Clinical features of patients infected with 2019 novel coronavirus in Wuhan, China. Lancet. 2020;395(10223):497-506.
2. Yang Y, Shang W, Rao X. Facing the COVID-19 outbreak: what should we know and what could we do? J Med Virol. 2020.

3. The Lancet. COVID-19: fighting panic with information. Lancet. 2020;395(10224):537.

4. Raptis CA, Hammer MM, Short RG, Shah A, Bhalla S, Bierhals AJ, et al. Chest CT and coronavirus disease (COVID-19): a critical review of the literature to date. AJR Am J Roentgenol. 2020;16:14.

5. Guedj E, Verger A, Cammilleri S. PET imaging of COVID-19: the target and the number. Eur J Nucl Med Mol Imaging 202017.

6. Juengling FD, Maldonado A, Wuest F, Schindler TH. The role of nuclear medicine for COVID-19 - time to act now. J Nucl Med 202017.

7. Tulchinsky M, Fotos JS, Slonimsky E. Incidental CT findings suspicious for Covid-19 associated pneumonia on nuclear medicine exams: recognition and management plan. Clin Nucl Med 20209.

8. Lütje S, Marinova M, Kütting D, Attenberger U, Essler M, Bundschuh RA. Nuclear medicine in SARS-CoV-2 pandemia: 18F-FDG-PET/CT to visualize COVID-19. Nuklearmedizin 2020 7.

9. Zuckier LS, Gordon SR. COVID-19 in the nuclear medicine department, be prepared for ventilation scans as well! Nucl Med Commun. 2020;41(5):494-5.

10. Polverari G, Arena V, Ceci F, Pelosi E, Ianniello A, Poli E, et al. (18)F-FDG uptake in asymptomatic SARS-CoV-2 (COVID-19) patient, referred to PET/CT for non-small cells lung cancer restaging. J Thorac Oncol. 2020.

11. Albano D, Bertagna F, Bertolia M, Bosio G, Lucchini S, Motta F, et al. Incidental findings suggestive OFCOVID-19 in asymptomatic patients undergoing nuclear medicine procedures in a high prevalence region. J Nucl Med 20201.

12. Zuckier LS, Moadel RM, Haramati LB, Freeman L. Diagnostic evaluation of pulmonary embolism during the COVID-19 pandemic. J Nucl Med 20201.

13. Deng Y, Lei L, Chen Y, Zhang W. The potential added value of FDG PET/CT forCOVID-19 pneumonia. Eur J Nucl Med Mol Imaging 202021.

14. Zou S, Zhu X. FDG PET/CT of COVID-19. Radiology 2020 6: 200770.

15. Qin C, Liu F, Yen TC, Lan X. (18)F-FDG PET/CT findings of COVID-19: a series of four highly suspected cases. Eur J Nucl Med Mol Imaging. 2020;47(5):1281-6.

16. Lam WW, Loke KS, Wong WY, Ng DC. Facing a disruptive threat: how can a nuclear medicine service be prepared for the coronavirus outbreak 2020? Eur J Nucl Med Mol Imaging. 2020.

17. Zhang X, Shao F, Lan X. Suggestions for safety and protection control in Department of Nuclear Medicine during the outbreak of COVID-19. Eur J Nucl Med Mol Imaging 202025.

18. Czernin J, Fanti S, Meyer PT, Allen-Auerbach M, Hacker M, Sathekge M, et al. Imaging clinic operations in the times of COVID-19: strategies, precautions and experiences J Nucl Med 20201.

19. Assadi M, Gholamrezanezhad A, Jokar N, Keshavarz M, Picchio $\mathrm{M}$, Seregni E, et al. Key elements of preparedness for pandemic coronavirus disease 2019 (COVID-19) in nuclear medicine units. Eur J Nucl Med Mol Imaging 202021.

20. Paez D, Gnanasegaran G, Fanti S, Bomanji J, Hacker M, Sathekge $\mathrm{M}$, et al. COVID-19 pandemic: guidance for nuclear medicine departments. Eur J Nucl Med Mol Imaging 202015.

21. Lu Y, Yan SX, Lan X, Zhu X, Macapinlac HA. Nuclear medicine in responding to global pandemic COVID-19-American College of Nuclear Medicine member experience. Eur J Nucl Med Mol Imaging 202015.

22. Loke KSH, Tham WY, Bharadwaj P, Keng FYJ, Huang Z, Idu MB, et al. Adapting to a novel disruptive threat: nuclear cardiology 
service in the time of the coronavirus (COVID-19) outbreak 2020 (SARS REBOOT). J Nucl Cardiol 202019.

23. Buscombe JR, Notghi A, Croasdale J, Pandit M, O’Brien J, Graham $\mathrm{R}$, Council and Officers of British Nuclear Medicine Society, et al. COVID-19: guidance for infection prevention and control in nuclear medicine. Nucl Med Commun. 2020.

24. Bebbington NA, Haddock BT, Bertilsson H, Hippeläinen E, Husby EM, Tunninen VI, et al. A Nordic survey of CT doses in hybrid PET/CT and SPECT/CT examinations. EJNMMI Phys. 2019;6(1): 24.

25. Poli GL, Torres L, Coca M, Veselinovic M, Lassmann M, Delis H, Fahey F. Paediatric nuclear medicine practice: an international survey by the IAEA. Eur J Nucl Med Mol Imaging 20198.

26. Wu D, Gomes Lima CJ, Bloom G, Burman KD, Wartofsky L, Van Nostrand D. Nationwide survey on implementation of 2011 nuclear regulatory commission policy on release of patients after (131)I therapy for thyroid cancer. J Nucl Med. 2020;61(3):397-404.

27. Dickson JC. Quantitative SPECT: a survey of current practice in the UK nuclear medicine community. Nucl Med Commun. 2019;40(10):986-94.
28. Zukotynski KA, Niederkohr RD, Greenspan BS, Prior JO, Schöder $\mathrm{H}$, Seltzer MA, et al. An international survey of PET/CT clinical reporting. J Nucl Med. 2019;60(4):478-9.

29. Halkar RK, Taylor AT. Survey on the use of nuclear renal imaging in the United States. J Nucl Med Technol. 2017;45(1):65.

30. Fendler WP, Czernin J, Herrmann K, Beyer T. Variations in PET/ MRI operations: results from an international survey among 39 active sites. J Nucl Med. 2016;57(12):2016-21.

31. Nishiyama Y, Kinuya S, Kato T, Kayano D, Sato S, Tashiro M, et al. Nuclear medicine practice in Japan: a report of the eighth nationwide survey in 2017. Ann Nucl Med. 2019;33(10):725-32.

32. Freudenberg LS, Dittmer U, Herrmann K. Impact of COVID-19 on nuclear medicine in Germany, Austria and Switzerland: an international survey in April 2020.Nuklearmedizin. 2020 Apr 28.

33. Treglia G. The role of (18)F-FDG PET for COVID-19 infection: myth versus reality. Clin Transl Imaging. 2020;30:1-2.

Publisher's note Springer Nature remains neutral with regard to jurisdictional claims in published maps and institutional affiliations. 